\title{
Comportements mécaniques sous indentation
}

\author{
Hervé Pelletier ${ }^{1}$ et Eric Le Bourhis ${ }^{2}$
}

Du 10 au 12 décembre 2014, à l'institut Charles Sadron (UPR 22 CNRS, Strasbourg), s'est tenu le colloque Indentation 2014, deuxième édition de ce congrès francophone à la suite de celui de 2012 qui avait été organisé par le laboratoire LTDS à l'Ecole Centrale de Lyon. Le colloque a réuni tous les scientifiques utilisateurs avancés des techniques d'indentation instrumentée. Ces techniques d'indentation statique ou dynamique (rayure) sont de plus en plus utilisées à l'échelle industrielle comme outil de caractérisation des surfaces de matériaux fonctionnalisés. Ces techniques récentes ne sont pas encore au stade des essais conventionnels de caractérisation des matériaux massifs (essais de traction, essais de compression ou encore de cisaillement). C'était justement l'objet du colloque Indentation 2014 : faire un état des lieux des recherches fondamentales (compréhension de la mécanique complexe des essais d'indentation et de rayure) et appliquées (informations déduites des essais sur des matériaux nouveaux ou innovants) en cours dans le domaine.

Durant ce colloque, qui a réuni 70 participants issus des équipes françaises possédant des équipements instrumentaux et/ou des moyens numériques dédiés aux techniques d'indentation, 25 présentations orales et environ une vingtaine de posters ont été présentés. L'ensemble de ces travaux présentés, lors de 5 sessions a suscité de nombreuses discussions et échanges entre les participants. Deux conférenciers Mr. Stéphane André (LEMTA) et Mme Trunfio Sfarghiu (LaMCoS) ont été invités pour présenter respectivement les enjeux de la métrologie contemporaine et la spectroscopie de forces atomiques pour l'analyse mécanique et tribologique des contacts biologiques.

Nous présentons dans ce numéro thématique, un ensemble d'articles issus d'une sélection de contributions au colloque, publiés après un processus d'évaluation et de révision. Ces articles offrent un aperçu de toutes les thématiques abordées au cours du colloque. L'ensemble du numéro regroupe des travaux fondamentaux et appliqués et permet de faire un point sur des sujets d'actualité dans le domaine de l'indentation. Le lecteur y trouvera des travaux couvrant un large spectre de matériaux. Différentes analyses des comportements élastique, fragile et ductile investiguées aux échelles pertinentes sont présentées et discutées.

Les éditeurs (H. Pelletier et E. Le Bourhis) tiennent à remercier les membres du comité d'organisation (A. Rubin, V. Le Houerou, J. Krier, T. Roland, L. Jacomine, D. Favier, H. Pelletier, C. Gauthier) pour la tenue de ce colloque, ainsi que les membres du comité scientifique du groupe indentation multiéchelle (E. Barthel, S. Benayoun, D. Chicot, J.-P. Guin, V. Keryvin, E. Le Bourhis, J.-L. Loubet, G. Mauvoisin, H. Pelletier, http://www.sf2m.asso.fr/ CommissionsThematiques/Indentation.htm) qui ont accepté de rapporter chacun sur plusieurs articles publiés dans cet ouvrage.

\footnotetext{
1 Institut Charles Sadron, UPR 22 CNRS, 23 rue du Loess, BP 84047, 67034 Strasbourg, France

2 Institut P', UPR 3346, CNRS-Université de Poitiers, Département de Physique et Mécanique des Matériaux, SP2MI, Boulevard Marie et Pierre Curie, BP 30179, 86962 Futuroscope Chasseneuil Cedex, France

Eric.le.bourhis@univ-poitiers.fr
} 


\section{Programme du congrès Indentation 2014}

\section{Session 1 : Mécanique de l'essai pour la caractérisation des matériaux}

G. Guillonneau, J. Michler

Détermination de l'aire de contact projetée réelle sous charge par nanoindentation in-situ

L. Rabemananjara, G. Mauvoisin, X. Hernot, A. Gavrus, J.M. Collin

Formulation de déformation représentative et de vitesse de déformation représentative par indentation conique

M. Yetna N'jock, D. Chicot, J.M. Ndjaka, J. Lesage, X. Decoopman, F. Roudet, A. Mejias

Un critère simple d'identification du mode de déformation par indentation

L. Charleux, V. Keryvin, J. Guin, M. Nivard, J-C. Sanglebøeuf

Accurate measurement of the indentation projected contact area on glasses

A. Rubin, D. Favier, C. Gauthier

Analyse in situ de contact viscoélastique sur polymère vitreux

G. Kermouche, A. Tanguy, J. Teisseire, G. Guillonneau, J. Michle, E. Barthel

Plastic flow of silica under uniaxial micro-compression - in situ investigation and multiscale modeling

B. Passilly, V. Jardret, M. Fajfrowski

Développements récents en indentation à $1000{ }^{\circ} \mathrm{C}$

J. Nohava, M. Conte, B. Bellaton, G. Berthout

Development and the first experiments with a new high temperature nanoindentation device

\section{Session 2 : Indentation pour la caractérisation de matériaux innovants}

M. Laurent-Brocq, L. Perrière, Y. Champion

La nanoindentation au service de l'étude de nouveaux concepts de matériaux

J-L. Loubet, J. Thevenet, P. Sotta, C. Fayolle, L. Vanel

Indentation et usure d'élastomeres charges réticulés

V. Le Houérou, F. Morestin, C. Gauthier, M-C. Baietto

Frottement dans les contacts textures de matière molle : étude locale par corrélation d'images

M. Buisson, T. Rouxel

Examen du modèle d' « ampoule » de E. Yoffe.

N. Le Roux, S. Meille, C. Langlois, T. Douillard, I. Zacharie-Aubrun, J.M. Gatt

Détermination de paramètres de rupture par indentation sur céramiques

G. Arnold, C. Auray, A. Giraud, G. Armand

Analyse par grille d'indentations du comportement mécanique d'argilite à température et hygrométrie controlées

\section{Session 3 : Indentation pour la caractérisation de films minces}

F. Roudet, D. Chicot, X. Decoopman, A. Iost, J. Bürgi, J. García Molleja, J. Feugeas

Propriétés mécaniques par indentation d'un film mince nanométrique de nitrure d'aluminium

O. Baudino, M. Saadaoui, D. Presle, B. Arrazat, K. Inal, B. Dubois

Détermination de la dureté de films poreux à nano domaine sur substrat flexible pour l'optimisation de son interconnexion avec une puce

H. Pelletier, D. Favier, C. Gauthier

Fatigue de surfaces de polymères revetues

F. Guillemot, J. Teisseire, E. Barthel, J.-P. Boilot, T. Gacoin

Couches minces macroporeuses et nanoindentation comment déjouer la conspiration?

R. Coq Germanicus, S. Eve, F. Lallemand, E. Hug

Étude multi-echelle de la nanoindentation du $\mathrm{Si}_{3} \mathrm{~N}_{4}$ : influence de la sous-couche de passivation des composants microélectroniques

N. Azeggagh, L. Joly-Pottuz, D. Nélias, J. Chevalier, T. Hashida

Détermination des paramètres rhéologiques des nitrures de silicium par méthode inverse 


\section{Session 4 : Rayures et essais multicontact}

C.H. Sacre, Ph. Guaino, L. Libralesso, A. Favache, T. Pardoen

Caractérisation et modélisation des mécanismes de rayure dans des films durs et fragiles deposés sur sous-couche compliante

T. Bourrel, V. LeHouérou, J. Krier, C. Gauthier

Mise en place de critères d'endommagement lors de la rayure de systèmes nus et revêtus par simulation numérique

H. Al baida, G. Kermouche, C. Langlade, J-F. Carton

Identification du comportement mécanique des matériaux à partir des essais de micro-impact répétés

\section{Session 5 : IndentationIdentifiabilité pour la caractérisation de matériaux métalliques et tôles}

F. Richard, R. Ben Hmida, S. Thibaud

Identifiabilité des paramètres d'une loi élastoplastique endommageable d'une tôle mince : indentation versus formage incrémental

M. Idriss, O. Bartier, X. Hernot, G. Mauvoisin, E. Gazo-Hanna

Caractérisation par indentation de tôles épaisses en alliage d'aluminium et de tôles minces en acier

E. Renner, Y. Gaillard, F. Richard, P. Delobelle

Analyse expérimentale et numérique de l'anisotropie et de la plasticité cristalline d'un polycristal de Ni par nanoindentation

\section{Posters}

A. Mejias, D. Chicot, X. Decoopman, A. Montagne, F. Roudet, A. Iost

Multi-scale approach of the instrumented indentation technique on the fracture toughness estimation

D. Mercier, C. Zambaldi, Y. Su, P. Eisenlohr, M. A. Crimp et T. R. Bieler

Caractérisation par indentation sphérique et modélisation de la plasticité cristalline près d'un joint de grain dans un échantillon de a -Ti pur.

E. Le Bourhis, K. Pantzas, G. Patriarche, A. Itawi, G. Beaudoin, I. Sagnes, A. Talneau, D. Troadec

Comportement mécanique de nanostructures Inp/Si

Kh. Ayadi et co-auteurs

Effets des traitements thermiques sur les contraintes et les fissures d'indentation

V. Keryvin, L. Charleux, J-P. Guin, M. Nivard, J-C. Sanglebœuf

Mécanismes de déformation des verres silicates sous chargement hydrostatique et lien avec leur réponse en nanoindentation

M. Laurent-Brocq, E. Béjanin, Y. Champion

Influence de l'état de surface sur les mesures de nanoindentation

D. Tumbajoy-Spinel, G. Kermouche, S. Descartes, J.M. Bergheau, G. Guillonneau, J. Michler

Identification des propriétés mécaniques des surfaces tribologiquement transformées par essais nano-mécaniques

E. S. Puchi-Cabrera, M. H. Staia, A. Iost

Modeling the composite hardness of coated systems involving multilayer coatings

C. Valantin, F. Lacroix, M.P. Deffarges, J. Morcel, Denis Tricoche, N. Ä̈t Hocine.

Evolution en fatigue des propriétés mécaniques à l'interface textile/caoutchouc

L. Jacomine, V. Le Houérou, C. Gauthier.

Le rôle des conditions environnementales sur les propriétés mécaniques de matériaux polymères.

N. Sheiko, Y. Holl, L. Jacomine, V. Le Houérou, C. Gauthier, P. Kékicheff

Effect of relative humidity on friction properties of nitrocellulose films

N. Yi, A. Rubin, T. Roland, C. Gauthier

Contact mechanics, friction and contact fatigue on polymeric surfaces 
H. Pelletier et E. Le Bourhis : Matériaux \& Techniques 103, 601 (2015)

L. Fu, A. Rubin, D. Favier, T. Charitat, C. Gauthier

Mesure du cisaillement de couches phospholipides confinées par recouvrement de fluorescence après photoblanchiment périodique (FRAPP)

A. Al-Kawaz, A. Rubin, I. Janowska, C. Pham-Huu, P. Mesini, C. Gauthier

Preparation and tribological properties of pmma/graphene composites coating layers

G. Arnold, N. Lauridant, T. J. Daou, D. Faye, M.-H. Tuilier, J. Patarin

Films zéolithiques sur alliage d'aluminium 7075 : propriétés mécaniques du revêtement et du substrat

M. Pissinier, A. Van Gorp, A. Montagne, A. Iost

Interférométrie appliquée à la réduction des erreurs en rupture par indentation

C. Moussa, G. Mauvoisin, O. Bartier, X. Hernot, G. Delattre

Caractérisation des matériaux métalliques par indentation sphérique : utilisation d'une déformation représentative pour plusieurs profondeurs d'indentation

C. Moussa, G. Mauvoisin, O. Bartier, X. Hernot, G. Delattre

Caractérisation d'aciers carbonitrures avec l'indentation sphérique 